\title{
CONDUTA NUTRICIONAL PARA PREVENÇÃO E TRATAMENTO DA ATEROSCLEROSE
}

\section{CONDUCTA NUTRICIONAL PARA LA PREVENCIÓN Y TRATAMIENTO DE LA AEROSCLEROSIS}

\section{NUTRITIONAL CONDUCT FOR THE PREVENTION AND TREATMENT OF AEROSCLEROSIS}

Autor 1: Victor de Oliveira Santos

Nutricionista Clínico ORCID: https://orcid.org/0000-0002-9070-2801 E-mail: victorssbm0111@ hotmail.com

Autor 2: Regiane Izabel de Oliveira Dias.

Nutricionista ORCID: https://orcid.org/0000-0001-8682-6943

E-mail: regianedias070@gmail.com

Autor 3: Jucimara Martins dos Santos Nutricionista Clínica do Centro do Idoso da Pref. Municipal de Barra Mansa, Professora Universitária do Centro Universitário de Barra Mansa (UBM) dos Cursos de Nutrição, Enfermagem, Fisioterapia e Educação Física. Mestre em Ciência pela UFRRJ

Barra Mansa - RJ - Brasil https://orcid.org/0000-0001-5121-6061 E-mail: : jucimara_martins@yahoo.com.br 
SANTOS, Victor et al. "Conduta Nutricional para Prevenção e Tratamento da Aterosclerose" ?

R. Científica UBM - Barra Mansa (RJ), ano XXVI, v. 23, n. 45, 2 . Sem. 2021 p. 98-110. ISSN 1516-4071

\section{RESUMO}

A aterosclerose é uma doença de patogenia lenta, progressiva e irreversível, decorrente de vários fatores, citando a dislipidemia. Trata-se de uma lesão do endotélio devido ao depósito de gordura, LDL-colesterol oxidado, células de espuma, cálcio e fibrina nos revestimentos internos da parede arterial, resultando em um processo inflamatório crônico. A morte por doenças cardiovasculares é a principal causa de morte no mundo, sendo a aterosclerose a patogenia frequentemente associada. Este trabalho teve como objetivo abordar a conduta nutricional para prevenção e tratamento da aterosclerose, além de descrever a patologia, os fatores de risco para a formação das placas de ateroma, apresentar os aspectos para diagnóstico da doença e descrever a recomendação dietoterápica para pacientes ateroscleróticos. Trata-se de uma revisão das literaturas nacionais disponíveis nas plataformas NCBI, Google Acadêmico, Scielo e Cochrane. É possível concluir que a adoção de novos hábitos alimentares e estilo de vida saudável são fatores importantes para tratamento e prevenção da doença

\section{Palavras-Chave: Aterosclerose. Doença Cardiovascular. Nutrição.}

\section{RESUMEN}

La aterosclerosis es una enfermedad de patogénesis lenta, progresiva e irreversible, resultante de varios factores, incluida la dislipidemia. Es una lesión en el endotelial debida al depósito de grasa, colesterol LDL oxidado, células espumosas, calcio y fibrina en el revestimiento interno de la pared arterial, lo que resulta en un proceso inflamatorio crónico. La muerte por enfermedad cardiovascular es la principal causa de muerte en todo el mundo, siendo la aterosclerosis la patogenia asociada con frecuencia. Este trabajo abordará la conducta nutricional para la prevención y tratamiento de la aterosclerosis, además de describir la patología, abordando los factores de riesgo para la formación de placas ateromatosas; presentar los aspectos para el diagnóstico de la enfermedad y describir la recomendación dietética para pacientes ateroscleróticos. Es una revisión de la literatura nacional disponible en las plataformas NCBI, Google Scholar, Scielo y Cochrane. Es posible concluir que la adopción de nuevos hábitos alimentarios y estilos de vida son un factor importante para el tratamiento y la prevención.

Palavras Clave: Aterosclerosis. Enfermedade Cardiovascular. Nutrición.

\section{ABSTRACT}

Atherosclerosis is a disease of slow, progressive and irreversible pathogenesis, resulting from several factors, including dyslipidemia. It is an endothelial injury due to fat deposits, oxidized LDL-cholesterol, foam cells, calcium and fibrin in the inner lining of the arterial wall, resulting in a chronic inflammatory process. Death from cardiovascular disease is the leading cause of death worldwide, with atherosclerosis being the pathogenesis frequently associated. This work will address the nutritional conduct for prevention and treatment of atherosclerosis, in addition to describing the pathology, addressing the risk factors for the formation of atheromatous plaques; present the aspects for diagnosis of the disease and describe the diet therapy recommendation for atherosclerotic patients. It is a review of national literature available on the NCBI, Google Scholar, Scielo and Cochrane platforms. It is possible to conclude that the adoption of new eating habits and lifestyle are an important factor for treatment and prevention

Keywords: Atherosclerosis. Cardiovascular Diseases. Nutrition. 


\section{INTRODUÇÃO}

A doença cardiovascular (DCV) constitui um grupo de doenças inter-relacionadas que incluem aterosclerose, hipertensão arterial (HA), doença cardíaca isquêmica, doença vascular periférica e insuficiência cardíaca (IC) (MAHAN \& RAYMOND, 2018). Costa \& Mendonça in Cuppari (2009) afirmam que as doenças cardiovasculares (DCV) representam a principal causa de morte em países desenvolvidos e em desenvolvimento, como o Brasil. Os autores apresentam, ainda, vários estudos que demonstraram o indiscutível papel das dislipidemias (LDL elevada e HDL diminuída), hipertensão arterial sistêmica (HAS), fumo, idade e diabete melito (DM) como fatores de risco independentes para o desenvolvimento da aterosclerose e suas complicações. Brasil, (2018) corrobora estes estudos, estimam que 17,7 milhões de pessoas morreram por doenças cardiovasculares em 2015, representando $31 \%$ de todas as mortes em nível global. Desses óbitos, estima-se que 7,4 milhões ocorrem devido as doenças cardiovasculares e 6,7 milhões devido a acidentes vasculares cerebrais.

A patogenia mais encontrada nas doenças cardiovasculares é a aterosclerose, sendo originada por disfunção endotelial e inflamação (BRASIL, 2018).

A aterosclerose é caracterizada pela lesão do endotélio, ocasionando o depósito de gordura, LDL-colesterol oxidado, células de espuma, cálcio e fibrina nos revestimentos internos da parede arterial, provocando estreitamento do canal sanguíneo que oxigenam o músculo cardíaco. A placa, aterosclerose, pode sofrer ruptura causando a formação de um coágulo sanguíneo que bloqueia a artéria ou outra parte do corpo por onde circular, por esse motivo, há possibilidade de ocorrer o infarto agudo do miocárdio (IAM), ou acidente vascular encefálico (AVE) (WILLIAMS, 2002; MAHAN \& RAYMOND, 2018).

Há evidências de que modificações do estilo de vida e redução de fatores de risco podem retardar o desenvolvimento da doença coronariana e outras formas de manifestação da aterosclerose. (COSTA \& MENDONÇA in CUPPARI, 2009; MAHAN \& RAYMOND, 2018).

A alimentação exerce grande importância na prevenção e no tratamento da aterosclerose, sendo o nutricionista responsável pelo acompanhamento alimentar do paciente, associado à equipe médica, garantindo o controle de peso, diminuição de gordura trans da dieta, redução no consumo de sal e introdução de alimentos funcionais e hábitos saudáveis. 
A obesidade e a dislipidemia resultam em aumento dos níveis séricos de LDL e LDL oxidadas, constituintes da placa de ateroma, e, portanto, é um fator de suma importância para a formação da placa aterosclerótica. Contudo, a obesidade e a dislipidemia são condições passíveis de mudança no estilo de vida, com uma reeducação alimentar adequada e monitoramento dos níveis lipídicos por meio de exames bioquímicos periódicos.

\section{FISIOPATOLOGIA DA ATEROSCLEROSE}

A aterosclerose (atero = gordura; esclerose $=$ fibrose) é um processo dinâmico, evolutivo e sistêmico que se desenvolve em artérias musculares médias e grandes (coronárias e artérias das extremidades inferiores) e em artérias elásticas (aorta, carótidas e ilíacas). Caracteriza-se pelo acúmulo de material lipídico no espaço intra e extracelular na camada íntima do endotélio, recoberto por capa fibrosa (COSTA \& MENDONÇA in CUPPARI, 2009).

A participação das lipoproteínas na evolução do processo aterosclerótico está bem estabelecida. A manutenção de níveis elevados de LDL e/ou níveis baixos de HDL está associada a um incremento na instabilização da placa. As lipoproteínas também têm ainda ações diretas sobre a coagulação: os quilomícrons ativam o fator VII; as VLDL aumentam a expressão de fibrinogênio e do inibidor da ativação do plasminogênio (PAI-1); as LDL promovem ativação plaquetária, pelo aumento do cálcio citosólico e expressão de prostaciclina e tromboxano A2; e as HDL promovem a inibição do fator tecidual. Reconhecida como um dos principais fatores de risco modificáveis para Doença Arterial Coronariana (DAC), as dislipidemias contribuem de modo significativo para o desenvolvimento da placa aterosclerótica e têm influência potencial na sua instabilização e expressão clínica (COSTA \& MENDONÇA in CUPPARI, 2009).

O plasma sanguíneo é constituído pelos integrantes lipídicos: colesterol livre, esterificado, triacilgliceróis (TG) e fosfolipídios que são ligados as proteínas formando partículas complexas chamadas de lipoproteínas para serem transportados (COZZOLINO, COMINETTI, 2013).

As lipoproteínas são classificadas em:

- Quilomícrons (Qm) (são partículas ricas em triglicérides, maiores e menos densas, sintetizadas nos enterócitos intestinais, responsáveis pelo transporte no plasma dos triglicérides originários da dieta e possuem Apo B48, Apo C e Apo E); 
SANTOS, Victor et al. "Conduta Nutricional para Prevenção e Tratamento da Aterosclerose" ?

R. Científica UBM - Barra Mansa (RJ), ano XXVI, v. 23, n. 45, 2 . Sem. 2021 p. 98-110. ISSN 1516-4071

- VLDL (apresentam muito baixa densidade, rica em triglicérides, de origem hepática e possui Apo B100, Apo E e Apo C);

- LDL (baixa densidade, rica em colesterol, transporta o colesterol do fígado para os tecidos periféricos e possui Apo B100);

- HDL (alta densidade, rica em colesterol, responsável pelo transporte reverso do colesterol, levando-o dos tecidos para o fígado e possui Apo A, Apo E e Apo C) (CUPPARI, 2014).

As dislipidemias são uma das maiores causas das doenças cardiovasculares, as Diretrizes relatam que os níveis séricos de Colesterol Total (CT) no Brasil, mostraram que $38 \%$ dos homens e $42 \%$ das mulheres tem níveis elevados (CT > $200 \mathrm{mg} / \mathrm{dL}$ ) neste caso, foram avaliadas 8.045 pessoas nas idades de 35 a 45 anos (CUPPARI, 2019).

A construção da placa aterosclerótica origina-se com a agressão ao endotélio vascular, como resultado o distúrbio endotelial da íntima às lipoproteínas plasmáticas, favorecendo a retenção destas no espaço subendotelial, e as partículas de LDL detidas sofrem oxidação causando a exposição de vários determinantes antigênicos, tornando-as imunogênicas. $O$ processo chave na formação de aterogênese ocorre com o depósito de lipoproteínas na parede arterial, outra ocorrência da disfunção endotelial é a chegada de moléculas de adesão leucocitária na superfície endotelial, responsáveis por atrair monócitos e linfócitos para o interior da parede arterial, processo estimulado pela presença de LDL oxidada (FALUDI et al, 2017).

Os macrófagos ativados em grande maioria são responsáveis pela evolução da placa aterosclerótica por meio da secreção de citocinas, que por sua vez amplificam a inflamação; e de enzimas proteolíticas, aptas de degradarem o colágeno e outros componentes teciduais locais (XAVIER et al, 2013). As LDL oxidadas agravam a lesão endotelial, resultando na liberação do fator de crescimento derivado das plaquetas (PDGF) que, por sua vez, estimula a proliferação e a migração das células musculares lisas para dentro da íntima (WIDH \& REINHARD, 2018). Além do aumento da produção de colágeno, a célula muscular lisa também produz as metaloproteinases, que são enzimas proteolíticas responsáveis pela degradação de colágeno, fibrina e elastina (MUTTONI, 2017). À medida que se produz a acumulação dos lipídios, a luz do vaso sanguíneo é progressivamente ocluída (GROPPER, 2011).

A placa aterogênica é formada por núcleo lipídico (rico em colesterol) e capa fibrosa (rica em colágeno), sendo caracterizadas em placas estáveis (domínio de colágeno, organizado 
em: capa fibrosa espessa, escassas células inflamatórias e núcleo lipídico de proporções menores) e instáveis (atividade inflamatória intensa, especialmente nas suas bordas laterais, com grande atividade proteolítica, núcleo lipídico proeminente e capa fibrótica amena) (CUPPARI, 2019).

\section{3 - FATORES DE RISCO PARA A GENESE DA ATEROSCLEROSE}

A OMS (2017) lista os mais importantes fatores de risco comportamentais, tanto para doenças cardíacas quanto para Acidente Vascular Cerebral (AVC), sendo eles: alimentação inadequada, sedentarismo, uso de tabaco e uso nocivo do álcool. Os efeitos dos fatores comportamentais de risco podem se manifestar em indivíduos por meio de pressão arterial elevada, glicemia alta, hiperlipidemia, sobrepeso e obesidade.

São conhecidos e comprovados alguns dos principais fatores de risco para DCV divididos em fatores de risco não modificáveis e modificáveis. Os fatores de risco não modificáveis são antecedentes familiares, sexo, idade avançada; e como fatores de risco modificáveis: HAS (hipertensão arterial sistêmica), o hábito de fumar, a inatividade física, o consumo de álcool em excesso, a obesidade e o sobrepeso, as dislipidemias, o diabetes mellitus (DM) e o estresse psicológico (CHAVES et al, 2015). Já é consenso que a redução dos fatores de risco diminui a DCV em indivíduos de todas as faixas etárias (MAHAN \& RAYMOND, 2018).

\section{4 - TRATAMENTO NUTRICIONAL DA ATEROSCLEROSE}

A terapia nutricional é a primeira conduta a ser adotada na prevenção e/ou tratamento das dislipidemias, em que o plano alimentar deverá contemplar questões culturais, regionais, sociais e econômicas, devendo ser agradável ao paladar e visualmente atraente. O paciente deverá receber também orientações relacionadas à seleção, às quantidades, técnicas de preparo e substituições dos alimentos (COSTA \& MENDONÇA in CUPPARI, 2009).

$\mathrm{O}$ aumento da prevalência da dislipidemia está fortemente associado às mudanças na alimentação, com predomínio de alimentos industrializados, que contribuem para a alteração do perfil lipídico e menor consumo de alimentos in natura. O profissional nutricionista deve indicar às famílias o consumo de alimentos saudáveis, fontes de vitaminas antioxidantes, principalmente frutas, verduras e legumes, por seu potencial efeito antioxidante (BONI et al, 2010). 
SANTOS, Victor et al. "Conduta Nutricional para Prevenção e Tratamento da Aterosclerose" ?

R. Científica UBM - Barra Mansa (RJ), ano XXVI, v. 23, n. 45, 2 . Sem. 2021 p. 98-110. ISSN 1516-4071

O National Cholesterol Education Program Expert Panel on Detection, Evaluation, and Treatment of High Blood Cholesterol in Adults (Adult Treatment Panel III, 2001) descreve que, entre as orientações de alimentação adequada para garantir a redução do risco de doença coronariana, encontra-se:

- $\quad$ Ingestão reduzida de gorduras saturadas como opção terapêutica para melhorar a redução do LDL,

- Optar por carboidrato complexos proveniente de alimentos ricos em fibras como grãos e cereais integrais, frutas e legumes.

- Evitar ácidos graxos trans, pois são outra gordura que aumenta o LDL colesterol.

\subsection{ALIMENTOS E MACRONUTRIENTES}

A partir de agora serão citados nutrientes e alimentos que auxiliam no tratamento como também na prevenção da aterosclerose.

\section{A) Fibra Dietética}

No grupo dos carboidratos complexos, têm-se as fibras dietéticas, que são classificadas de acordo com sua solubilidade, em solúveis e insolúveis. As fibras solúveis são representadas pela pectina (frutas) e pelas gomas (aveia, cevada e leguminosas: feijão, grão de bico, lentilha e ervilha). O farelo de aveia é o alimento mais rico em fibras solúveis e pode, portanto, diminuir moderadamente o colesterol sanguíneo (MONTES \& NASCIMENTO, 2013; MAHAN, RAYMOND, 2018).

A ingestão de fibra alimentar está inversamente correlacionada com vários fatores de risco para doenças cardiovasculares em ambos os sexos, o que sustenta seu papel protetor e recomendações para o aumento no consumo (LAIRON et al, 2005). Santos et al (2020) relatam vários estudos que corroboram a ação das fibras dietéticas; seu consumo dietético é proporcionalmente inverso a gênese de patologias, como diabete, patologias intestinais, obesidade e doença cardiovascular.

Para o propósito da prevenção da doença cardíaca, a maior parte da quantidade recomendada de 25 a $30 \mathrm{~g}$ de fibras por dia deve consistir em fibra solúvel. Os mecanismos propostos para o efeito hipocolesterolêmico das fibras solúveis incluem os seguintes:

1 - As fibras ligam-se aos ácidos biliares, o que diminui a concentração sérica de colesterol; 
2 - As bactérias no cólon fermentam as fibras para produzir acetato, propionato e butirato, os quais inibem a síntese de colesterol (MAHAN, RAYMOND, 2018).

B) Chá verde

O chá-verde contém polifenóis, catequinas e cafeína; possui propriedade antiinflamatória e é excelente estratégia para modular a inflamação e o estresse oxidativo. Os polifenóis do chá-verde auxiliam no controle do apetite, regulação negativa de enzimas, envolvendo metabolismo lipídico hepático e a diminuição da absorção de nutrientes sob a influência do sistema nervoso simpático (SANTOS et al, 2020).

Batista et al, (2008), realizou um estudo prospectivo, duplo cego e cruzado com o objetivo de investigar os efeitos do chá verde (Camellia sinensis) em pacientes portadores de dislipidemias. Participaram dessa pesquisa 33 pacientes, com idade entre 21 e 71 anos, que consumiam uma dieta com baixo teor de gorduras (25\% a 35\% das calorias totais e $200 \mathrm{mg}$ de colesterol por dia). Foram randomizados para dois tratamentos sequenciais: cápsulas contendo $250 \mathrm{mg}$ de extrato seco do chá verde ou placebo, administradas por um período total de 16 semanas, tendo cada paciente utilizado chá verde por oito semanas e placebo em igual período. As variações lipídicas médias, provocadas pelo uso do chá verde mostraram uma redução de 3,9 \% (p=0,006) nas concentrações do colesterol total e uma redução de 4,5 \% (p $=0,026)$ do LDL-colesterol. A ingestão de chá verde não influenciou significativamente os níveis de HDL-colesterol, dos triglicerídeos e do Apo-B. Resultados não significativos foram observados na avaliação dos lipídeos sanguíneos (colesterol total e LDL-colesterol) com o uso do placebo. Foi demonstrado o efeito benéfico do chá verde (Camellia sinensis), que reduziu significativamente, em oito semanas, os níveis de colesterol total e LDL-colesterol nesse grupo de pacientes.

\section{C) Linhaça}

A semente de linhaça possui alguns componentes que apresentam ações biológicas importantes, como o óleo com ômega 3 e fibras solúveis. Estudos atestam a capacidade preventiva e mesmo curativa da semente de linhaça sobre a aterosclerose (CARRARA et al, 2009)

Em um estudo randomizado, trinta coelhos foram separados em três grupos com base em um tratamento dietético por 12 semanas: grupo controle (G1), dieta padrão; dieta rica em colesterol (G2), dieta padrão mais $0,25 \%$ de colesterol proveniente de ovos liofilizados; e 
dieta rica em colesterol mais linhaça (G3), dieta semelhante à do G2 adicionada de linhaça. Foram realizadas em todos os grupos: análise bioquímica (colesterol total, HDL-colesterol, LDL-colesterol e triglicérides) e imunohistoquímica (molécula de adesão intercelular 1 [ICAM-1] e fator de necrose tumoral alfa [TNF- $\alpha]$ ). Após 12 semanas, os níveis séricos de CT aumentaram significativamente nos grupos G2 e G3. Os níveis séricos de LDL-colesterol foram mais altos no grupo G2, e o aumento no grupo G3 foi cerca de seis vezes menor do que no G2. Os níveis de HDL-colesterol aumentaram em todos os grupos, com o maior aumento observado no G2. Os níveis de triglicérides no G3 reduziram em $70 \%$ e diferiram significativamente entre o G1 e G3. Níveis de ICAM-1 aumentaram apenas no segmento aórtico 4 no G3. O grupo alimentado com linhaça mostrou redução no desenvolvimento de aterosclerose, níveis séricos mais baixos de triglicérides e níveis mais baixos de TNF- $\alpha$ à imunohistoquímica (MARTINS et al,2018).

D) Uva

A uva e seus derivados, como o vinho tinto, possuem compostos fenólicos, que são potentes antioxidantes e desempenham papel relevante na prevenção da oxidação da LDL-c e na redução de mediadores inflamatórios, atenuando, assim, o processo aterosclerótico. Estudos indicam que os polifenóis modulam a atividade de diversos fatores de transcrição, o que conduz à redução de citocinas inflamatórias e de moléculas de adesão e aumento da expressão de enzimas antioxidantes (SOUSA \& PEREIRA, 2013).

No estudo de Matos et al (2012), vinte coelhos brancos adultos do sexo masculino, foram selecionados e divididos em dois grupos: grupo controle (GC), 10 coelhos; e grupo resveratrol (GR), 10 coelhos. Os animais foram alimentados com uma dieta hipercolesterolêmica por 56 dias. Para a dieta do GR, o resveratrol $(2 \mathrm{mg} / \mathrm{kg}$ peso/dia) foi adicionado do $33^{\circ}$ ao $56^{\circ}$ dia. No GC, $70 \%$ apresentaram lesões ateroscleróticas avançadas da aorta (tipos III, IV, V ou VI). Todos os animais do GR apresentaram lesões ateroscleróticas leves da aorta (tipos I ou II) ou não apresentaram lesões. A razão entre a área intima e a área da camada intima/medial mostrou-se significativamente menor no GR quando comparada ao GC ( $p<0,001)$. Áreas positivas para moléculas de adesão celular vascular-1 (VCAM-1) foram menores no GR $(p=0,007)$. As concentrações de proteína quimiotática de monócitos-1 (MCP-1) e de interleucina-6 (IL-6) mostraram-se significativamente menores no GR do que no GC ( $p=0,039 \mathrm{e} p=0,015$, respectivamente). O Resveratrol apresentou importantes efeitos 
SANTOS, Victor et al. "Conduta Nutricional para Prevenção e Tratamento da Aterosclerose" ?

R. Científica UBM - Barra Mansa (RJ), ano XXVI, v. 23, n. 45, 2 . Sem. 2021 p. 98-110. ISSN 1516-4071

antiaterogênicos e anti-inflamatórios em um modelo animal com coelhos alimentados com uma dieta hipercolesterolêmica.

\section{E) Cúrcuma}

A curcumina é o ingrediente ativo do remédio fitoterápico tradicional e da cúrcuma (Curcuma longa)). A curcumina tem uma gama surpreendentemente ampla de propriedades benéficas, incluindo atividade anti-inflamatória, antioxidante, quimiopreventiva e quimioterápica (HATCHER H. et al, 2008 in SANTOS, 2020).

O estudo de SINGH, V. et al, (2013) investigou o potencial de modificação da doença com o uso de óleo de curcuma (C. oil), um componente lipofílico de C. longa L., em hamsters hiperlipidêmicos. Hamsters dourados sírios machos foram alimentados com ração ou colesterol alto (HC) e dieta rica em gordura com ou sem óleo de cúrcuma (30, 100 e 300 mg / $\mathrm{kg}$ ) por 28 dias. Em hamsters HC alimentados com dieta, o C. oil reduziu significativamente o colesterol total plasmático, o colesterol LDL e o TAG, e aumentou o colesterol HDL quando comparado com o grupo HC. Comparações de grupos semelhantes mostraram que o tratamento com óleo de cúrcuma reduziu o colesterol hepático e o estresse oxidativo e melhorou a função hepática. Ativação plaquetária induzida por hiperlipidemia, disfunção vascular e expressão reprimida de mRNA de eNOS foram restauradas pelo tratamento com $C$. oil. Além disso, o acúmulo de colesterol aórtico e a expressão de CD68 também foram reduzidos no grupo tratado com óleo. O efeito anti-hiperlipidêmico exibido por $C$. oil parece ser mediado pela modulação de PPARa, LXRa e genes associados envolvidos no metabolismo e transporte de lipídeos.

\section{F) Cebolinha Francesa}

As espécies Allium são cultivadas para o bulbo comestível, que é usado principalmente como aromatizante em alimentos. Além disso, pode prevenir a promoção do tumor e alguns processos que estão associados aos radicais livres, como doenças cardiovasculares e envelhecimento (TAJNER et al, 2008).

No estudo de Alves et al (2019) com o objetivo de analisar os efeitos do consumo do chá de cebolinha francesa (Allium schoenoprasum L.) no colesterol total (CT) e nos triglicerídeos (TG) de camundongos, recém desmamados, submetidos à dieta hiperlipídicautilizaram-se 40 animais, os quais foram distribuídos aleatoriamente em 4 grupos com 10 
animais cada. O grupo 1 e 2 receberam água e chá, respectivamente, junto com ração padrão. Os grupos 3 e 4 receberam água e chá, respectivamente, com dieta hiperlipídica. Por fim, realizou-se análise estatística nos resultados para verificação do nível de influência dos resultados. Houve diferença significativa no colesterol total e nos triglicerídeos dos animais os quais consumiram dieta hiperlipídica. Notou-se também, uma redução significante de colesterol e triglicerídeos dentre os animais os quais consumiram o chá de cebolinha francesa. A dieta hiperlipídica apresentou hiperlipidêmico, todavia, o consumo de chá de cebolinha mostrou-se eficaz no controle do colesterol total e triglicerídeos.

\section{CONSIDERAÇÕES FINAIS}

A aterosclerose é uma doença multifatorial, silenciosa e progressiva, que tem afetado a população do mundo todo. A sua morbidade e mortalidade se refletem nos índices elevados das doenças cardiovasculares, as quais lideram as principais causas de óbito no mundo. Por ser uma patologia multifatorial, o acompanhamento multidisciplinar se faz indispensável.

Sabendo do caráter inflamatório da aterosclerose, como também do excesso de peso, a adoção de hábitos alimentares saudáveis é inevitável para garantir a saúde. Assim como a inclusão de alimentos funcionais na dieta capazes de auxiliar no tratamento e prevenção da doença. Portanto, a reeducação alimentar é parte importante do tratamento e da prevenção da doença. Manter os indicadores sanguíneos do lipidograma em valores adequados é fundamental. A boa nutrição, com orientação profissional, e um estilo de vida saudável, são fundamentais.

Dada a importância do tema, compreende-se como necessário o desenvolvimento de novos estudos capazes de descrever uma variedade maior de alimentos funcionais para o tratamento da doença.

\section{REFERÊNCIAS}

ALVES, J. N. C. A. et al. Efeitos do consumo de chá de cebolinha francesa (Allium schoenoprasum L.) no colesterol e triglicerídeos de camundongos submetidos à dieta hiperlipídica. Seminário de Pesquisa e Iniciação Científica da UBM. - Área da Saúde / Centro Universitário de Barra Mansa. Rio de Janeiro: Publit, 2019. 
SANTOS, Victor et al. "Conduta Nutricional para Prevenção e Tratamento da Aterosclerose" ?

R. Científica UBM - Barra Mansa (RJ), ano XXVI, v. 23, n. 45, 2 . Sem. 2021 p. 98-110. ISSN 1516-4071

BATISTA, G.A.P. et al. A Camellia Sinensis nas dislipidemias. Arq Bras Cardiol.2009; 93(2): 128134.Disponível em: http://www.scielo.br/pdf/abc/v93n2/v93n2a10.pdf

BERNAUD, F.S.R.; RODRIGUES, T.C. Fibra alimentar - ingestão adequada e efeitos sobre a saúde do metabolismo. Arq Bras Endocrinol Metab.57/6. 2013. Disponível em http://www.scielo.br/pdf/abem/v57n6/01.pdf Acessado em 25/04/2020.

BONI, A. et al. Vitaminas antioxidantes e prevenção da arteriosclerose na infância. Revista Paulista de Pediatria. vol.28. no.4. SP. Oct./Dec. 2010. Disponível em: https://www.scielo.br/pdf/rpp/v28n4/a14v28n4.pdf

CARRARA, C.L.; ESTEVES, A.P.; GOMES, R.T.; GUERRA, L.L. Uso da semente de linhaça como nutracêutico para prevenção e tratamento da aterosclerose. Revista Eletrônica de Farmácia. Vol 6. N.4. pag. 1-9, 2009. Disponível em https://revistas.ufg.br/REF/article/view/8774/6115.

CHAVES, C. D. S. Identificação de fatores de risco para doenças cardiovasculares em profissionais da saúde. Arquivos de Ciências da Saúde.v. 22, n. 1, p. 39-46, mar. 2015. ISSN 2318-3691. Disponível em: 〈http://www.cienciasdasaude.famerp.br/index.php/racs/article/view/28>.

COZZOLINO, S.M.F.; COMINETTI C. Bases bioquímicas e fisiológicas da nutrição: nas diferentes fases da vida, na saúde e na doença. Barueri, São Paulo: Manole, 2013.

CUPPARI, L. et al. Nutrição clínica no adulto. 4ed. Barueri, São Paulo: Manole, 2019.

CUPPARI, L. et al. Nutrição: nas doenças crônicas não-transmissíveis. Barueri, São Paulo: Manole, 2009.

FALUDI, A.A. et al. Atualização da Diretriz Brasileira de Dislipidemias e Prevenção da Aterosclerose. Arq. Bras. Cardiol. 2017; 109(2Supl.1):1-76.

GROPPER, S.S.; SMITH, J.L.; GROFF, J.L. Nutrição avançada e metabolismo humano; tradução Marleine Cohen. $5^{\circ}$ ed. São Paulo: Cengage Learning, 2011.

HATCHER H., PLANALP, R.; CHO, J.; TORTI, F.M. Curcumin: From ancient medicine to current clinical trials. Cell. Mol. Life Sci. 65, 1631-1652. 2008. Disponivel em: https://www.ncbi.nlm.nih.gov/pmc/articles/PMC4686230/.

LAIRON, D. et al. Dietary fiber intake and risk factors for cardiovascular disease in french adults. Jornal de Nutrição Clínica, 2005; 82 (6): 1185-1194. Disponível em: https://academic.oup.com/ajcn/article/82/6/1185/4648788.

MAHAN; L.K., RAYMOND J.L. KRAUSE alimentos, nutrição e dietoterapia; tradução Verônica Mannarino, Andréa Favano. 14 ${ }^{\circ}$ ed. Rio de Janeiro: Elsevier, 2018.

MARTINS, M. S. L. et al. Redução do Marcador Inflamatório TNF- $\alpha$ após Consumo de Semente de Linhaça por Coelhos Hipercolesterolêmicos. International Journal of Cardiovascular Sciences. 2018;31(2)114-122. Disponível em: https://www.scielo.br/pdf/ijcs/v31n2/pt_2359-4802-ijcs-31-020114.pdf

MATOS, R.S., Resveratrol provoca efeitos antiaterogênicos em um modelo animal de aterosclerose. Arq. Bras. Cardiol. vol.98 no.2 São Paulo Feb. 2012. Epub Jan 09, 2012. Disponível em: https://www.scielo.br/pdf/abc/v98n2/aop00112.pdf.

MUTTONI, S. Patologia da nutrição e dietoterapia. Porto Alegre: SAGAH, 2017. 
SANTOS, Victor et al. "Conduta Nutricional para Prevenção e Tratamento da Aterosclerose" ?

R. Científica UBM - Barra Mansa (RJ), ano XXVI, v. 23, n. 45, 2 . Sem. 2021 p. 98-110. ISSN 1516-4071

NATIONAL HEART, LUNG, AND BLOOD INSTITUTE. Third Report of the Expert Panel on Detection, Evaluation, and Treatment of High Blood Cholesterol in Adults (Adult Treatment Panel III). Disponível em: https://www.nhlbi.nih.gov/files/docs/resources/heart/atp-3-cholesterol-fullreport.pdf

NETO, A.A. et al. IV Diretriz Brasileira sobre Dislipidemias e Prevenção da Aterosclerose. Departamento de Aterosclerose da Sociedade Brasileira de Cardiologia. Arq. Bras. Cardiol. vol.88 suppl.1 São Paulo Apr. 2007

OMS - ORGANIZAÇÃO MUNDIAL DA SAÚDE. (2017, maio). Doenças cardiovasculares. Disponível em:

https://www.paho.org/bra/index.php?option=com_content\&view=article\&id=5253:doencascardiovasculares\&Itemid=1096. Acesso em:25 set.2020

ORGANIZAÇÃO MUNDIAL DA SAÚDE. (2017, maio). Doenças cardiovasculares. Disponível em: https://www.paho.org/bra/index.php?option=com_content\&view=article\&id=5253: doencascardiovasculares \&Itemid=1096. Acesso em:25 set.2020

SANTOS, J. M. et al. Ação dos alimentos funcionais no tratamento do processo inflamatório causado pela obesidade: uma revisão bibliográfica. R. Científica UBM - Barra Mansa (RJ), ano XXV, v. 22, n. 43, 2. $\quad$ Sem. $2020 \quad$ p. 23- 38. Disponível em: http://revista.ubm.br/plugins/generic/pdfJsViewer/pdf.js/web/viewer.html?file=http\%3A\%2F\%2Frevis ta.ubm.br\%2Findex.php \%2Frevistacientificaubm \%2Farticle $\% 2$ Fdownload $\% 2 \mathrm{~F} 733 \% 2 \mathrm{~F} 6 \% 2 \mathrm{~F}$

SINGH, V. et al. Curcuma oil ameliorates hyperlipidaemia and associated deleterious effects in golden Syrian hamsters. Br J Nutr. 2013 Aug 28;110(3):437-46. Epub 2013. Disponível em: https://pubmed.ncbi.nlm.nih.gov/23673139/.

SOUSA, S M.; PEREIRA A. C. M. Mecanismos moleculares de ação anti-inflamatória e antioxidante de polifenóis de uvas e vinho tinto na aterosclerose. Rev. Bras. Pl. Med., Campinas, v.15, n.4, p.617626, 2013.Disponível em: https://www.scielo.br/pdf/rbpm/v15n4/a20v15n4.pdf

STAJNER D. et al. Comparative study of antioxidant properties of wild growing and cultivated Allium species. Phytother Res. 2008 Jan;22(1):113-7. Disponível em: https://pubmed.ncbi.nlm.nih.gov/17726730/

WIDTH M.; REINHARD T. Manual de sobrevivência para nutrição clínica; tradução Carlos Henrique de A. Cosendey. $2^{\circ}$ ed. Rio de Janeiro: Guanabara Koogan, 2018.

WILLIAMS, M.H. Nutrição para saúde, condicionamento físico e desempenho esportivo. $1^{\circ}$ ed.Barueri, São Paulo:Manole,2002.

XAVIER H. T. et al. Sociedade Brasileira de Cardiologia. V Diretriz Brasileira de Dislipidemias e Prevenção da Aterosclerose. Arq Bras Cardiol, Volume 101, No 4, Suplemento 1, 2013. Disponível em: https://www.scielo.br/pdf/abc/v101n4s1/v101n4s1.pdf. 